Bull. Mater. Sci., Vol. 16, No. 2, April 1993, pp. 127-135. (C) Printed in India.

\title{
Spectroscopic, dark and photoconductive properties of some polyene-iodine charge-transfer complexes
}

\author{
DILIP GHOSH, SUSMITA HAZRA, PRABIR PAL and T N MISRA \\ Spectroscopy Department, Indian Association for the Cultivation of Science, Jadavpur, \\ Calcutta 700032, India
}

MS received 30 October 1992; revised 16 January 1993

\begin{abstract}
Spectroscopic evidence has been provided to confirm that zeaxanthin, lutein and fucoxanthin form molecular charge-transfer (CT) complexes with iodine in the solid state. The semiconductive and photoconductive properties of CT complexes have been investigated in polycrystals in a sandwich cell configuration. Both dark and photoconductivity increases by several orders of magnitude on complex formation. The identical values of thermal activation energies for dark and photoconduction have been obtained for the complexes and this has been attributed to spontaneous carrier generation by CT interaction and their migration by trapping and detrapping mechanisms. Photoconduction action spectra in pure polyene and in polyene-iodine complex suggest that photoinjection from the electrode and direct electron hole pair production are the two photocarrier generation mechanisms operative in these crystals. The second mechanism predominates in pure materials whereas the first one becomes important in the complexes.
\end{abstract}

Keywords. Dark and photoconduction; charge-transfer complexes; polyenes; zeaxanthin; lutein; fucoxanthin; organic semiconductor; organic photoconductor.

\section{Introduction}

In our earlier communications (Pal et al 1988; Pal and Misra 1989) we reported the photocarrier generation and their transport mechanism in a number of conjugated polyenes such as voltage, temperature and excitation light intensity dependence of the photocurrent and the photoconduction action spectra.

These biologically important materials are generally high resistance semiconductors and their conductivity is of the order of $10^{-14} \Omega^{-1} \mathrm{~cm}^{-1}$. However, when they form a complex with iodine, their conductivity goes as high as $10^{-6} \Omega^{-1} \mathrm{~cm}^{-1}$. Highly conducting organic charge-transfer complexes usually have segregated stacking of donors and acceptors and incomplete charge-transfer between the components is observed (Murata and Nakasuji 1985; Torrance 1985). The major features of most of the CT complexes are largely determined by $\Delta E_{\text {redox }}$, this being the difference between the electrochemical oxidation potential of the donor and the electrochemical reduction potential of the acceptor molecules. The large difference between the ionization potential $I_{D}$ of the donor and the electron affinity $E_{A}$ of the acceptor for the polyene-iodine system favour neutral type of complex formation. The stacking pattern of donors and acceptors in these lattices is not known. The mechanism for the big leap in conductivity in the polyene-iodine complexes is not understood. It was, therefore, thought worthwhile to extend our study to the dark and photoconductive properties of these CT complexes to have a better understanding of the carrier generation and their transport mechanism. In this paper we report our experimental results. We show here that the polyenes form molecular CT complexes with iodine and our results further confirm that the direct electron-hole pair production 
by irradiation is the principal mechanism of carrier generation in pure polyenes whereas photoinjection from electrode is dominant in CT complexes.

Trapping conduction seems to explain the activation energy of dark and photoconductivity in these CT complexes. However, the hopping mechanism cannot be ruled out (Tamamura et al 1974; Kivelson 1981).

\section{Experimental}

The polyenes used in the present investigation are zeaxanthin, lutein and fucoxanthin. The molecule structures are shown in figure 1. The samples, gifted by Hoffman-LaRoche, Basel, Switzerland, were of highly pure quality. The absorption spectra of these samples showed excellent agreement with the respective spectra reported in the literature for highly pure materials (Mark Index 1976). These were used without further purification. For absorption spectroscopy thin films of polycrystals of these polyenes were obtained on the quartz surface by gently rubbing the material on it. The solid films, thus obtained, were exposed to iodine vapour. The doping concentration of iodine increased with the length of exposure time. The absorption spectra were recorded on a Shimadzu spectrophotometer (model No. 210A) at room temperature $\left(27^{\circ} \mathrm{C}\right)$ immediately after exposure of the solid films to iodine vapour.

CT complexes were also prepared by placing the polyenes in powder form in iodine vapour in a desiccator at room temperature. The time of exposure was such that maximum doping optimization was achieved and details of this study have been discussed elsewhere (Sen et al 1992). The conductivity measurements were carried out using a sandwich cell with stainless steel and $\mathrm{SnO}_{2}$-coated glass electrodes separated by a teflon spacer of thickness $0.0025 \mathrm{~cm}$. The conducting glass was the positive<smiles>CC1=C(/C=C/C(C)=C/C=C/C(C)=C/C=C/C=C(C)/C=C/C=C(C)/C=C/C2=C(C)CC(O)CC2(C)C)C(C)(C)CC(O)C1</smiles>

Zeaxanthin<smiles>CC1=CC(O)CC(C)(C)C1/C=C/C(C)=C/C=C(C)/C=C(C)/C=C/C=C(C)/C=C/C=C(C)/C=C/C1=CCC(O)CC1(C)C</smiles>

Lutein

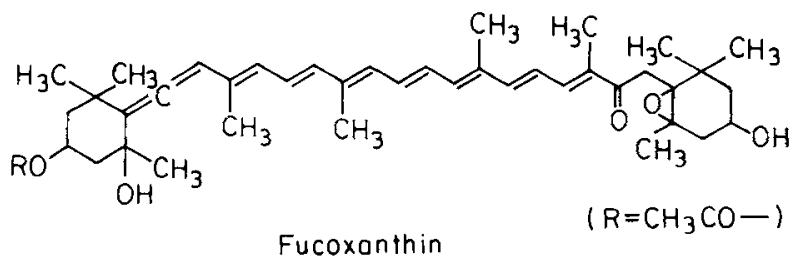

Figure 1. Molecular structure of some linear conjugated polyenes. 
electrode. Finely powdered samples sandwiched between the electrodes were kept at a moderate pressure of $400 \mathrm{~g} / \mathrm{cm}^{2}$ using spring clips. Adequate contact between the electrodes and powder material and also between the material particles was ensured by annealing through several heat-cool cycles. The cell was then placed in a suitably designed conductivity chamber (Mallik et al 1979) with a quartz window through which the sample was illuminated for photoconductivity study. A $100 \mathrm{~W}$ xenon lamp was used for steady state photoconductivity study. For current measurements Keithley's programmable electrometer (model No. 617) was used. This had a built-in isolated DC voltage source which can be adjusted between $-100 \mathrm{~V}$ to $+100 \mathrm{~V}$. DC voltages were applied across the electrodes from this source. Temperature was measured using copper-constantan thermocouple and panel meter (model No. 100, HIL, India). For photoconductivity action spectra measurements, a monochromator (Jobin Yvon No. H20 UV 1673, France) was used. The sandwich cell thickness and area were maintained at $0.0025 \mathrm{~cm}$ and $0.25 \mathrm{~cm}^{2}$ respectively. Each conductivity measurement was repeated at least five times on the same cell and the variations recorded were less than $2 \%$.

\section{Results and discussion}

\subsection{Electronic absorption spectra}

The electronic absorption spectra of zeaxanthin, lutein and fucoxanthin in the solid state and that after exposure to the iodine vapour have been studied. It was observed that on absorption of the acceptor vapour, a new band appears on the longer wavelength side of the spectrum in addition to the original band in each of these polyenes. Such spectra of zeaxanthin and lutein are shown in figures 2 and 3 respectively. The position of this new band depends on the polyene. The $\lambda_{\max }$ of this band was observed at 860,800 and $750 \mathrm{~nm}$ for zeaxanthin, lutein and fucoxanthin respectively. The intensity of the new band increased with increasing amount of acceptor vapour adsorbed on the donor film surface and ultimately reached maximum intensity after which there was no further change in peak height. This was the chargetransfer band of the molecular complexes of polyenes with iodine.

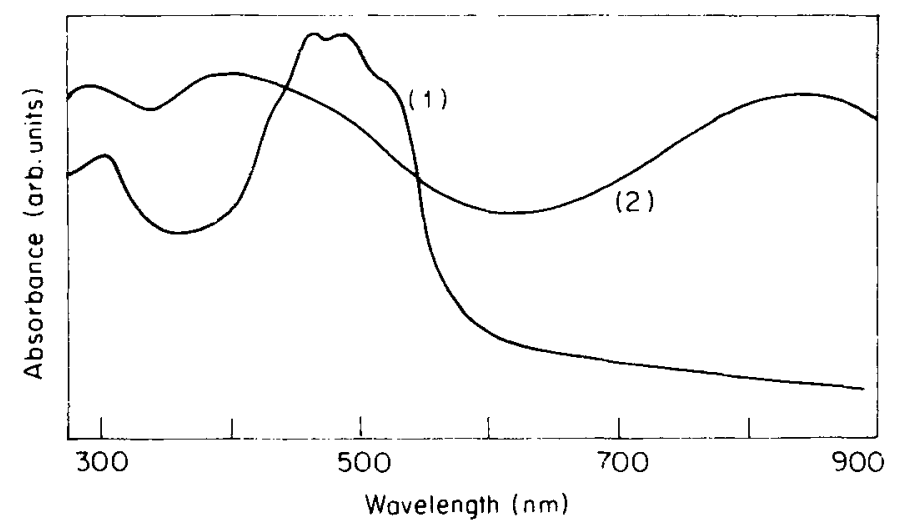

Figure 2. Absorption spectra for (1) zeaxanthin and (2) zeaxanthin-iodine complex. 


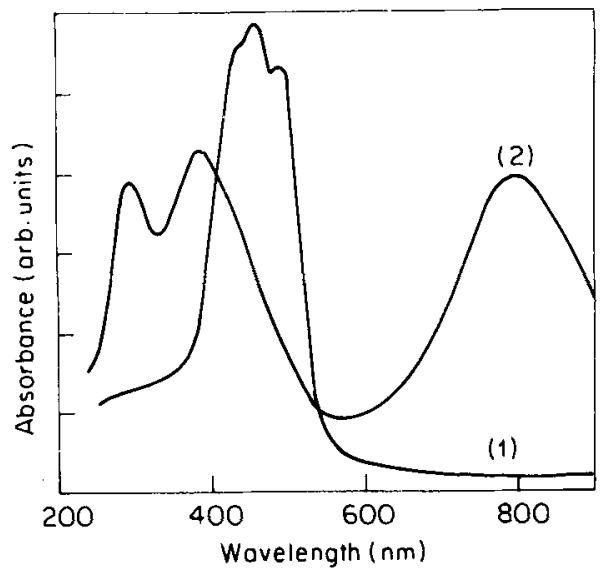

Figure 3. Absorption spectra for (1) lutein and (2) lutein-iodine complex.

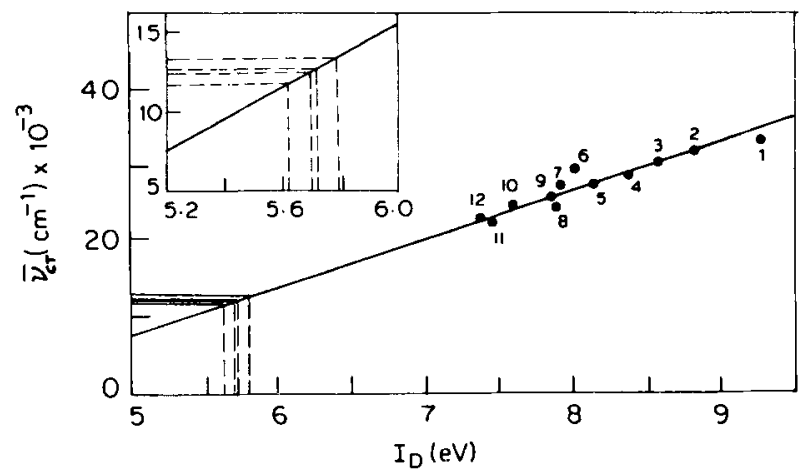

Figure 4. Plot of $\bar{v}_{\mathrm{CT}}$ against $I_{D}$ for CT complexes of number of donors with iodine acceptor. The donors are (1) benzene; (2) toluene; (3) m-xylene; (4) mesitylene; (5) napthalene; (6) durene; (7) pentamethylbenzene; (8) triphenylene; (9) hexamethylbenzene; (10) chrysene; (11) anthracene; and (12) Pyrene. (Values of $\bar{v}_{\mathrm{CT}}$ and $I_{D}$ have been taken from Chen and Wentworth $(1975)$ and Foster (1969). Evaluated $I_{D}$ values of polyenes are shown in the inset.

The charge-transfer band energy can be approximately related to the ionization potential of the donor and electron affinity of the acceptor molecule by the equation (Chen and Wentworth 1975).

$$
h v_{\mathrm{CT}}=I_{D}-E_{A}+C_{1}
$$

where $h$ is the Planck's constant, $v_{\mathrm{CT}}$, the frequency corresponding to the lowest energy intermolecular charge-transfer band, $I_{D}$, the vertical ionization potential of the donor, $E_{A}$, the vertical electron affinity of the acceptor and $C_{1}$, a constant.

The ionization potential of an unknown donor can be obtained from the $\bar{v}_{\mathrm{CT}}$ vs $I_{D}$ plot (figure 4) for a large number of other donors with a common acceptor, provided the value of $\bar{v}_{\mathrm{CT}}$ for the unknown donor is known for the same acceptor. The $\bar{v}_{\mathrm{CT}}$ and corresponding $I_{D}$ values were taken from literature (Foster 1969; Chen and Wentworth 1975; Mallik et al 1980).

The values of $I_{D}$ obtained from this plot for zeaxanthin, lutein and fucoxanthin 
Table 1. Ionization potential of polyenes.

\begin{tabular}{lccc}
\hline Polyenes & $\bar{v}_{\mathrm{CT}}\left(\mathrm{cm}^{-1}\right)$ & $\begin{array}{c}\text { Estimated } I_{D}(\mathrm{eV}) \\
\text { from }(2)\end{array}$ & $\begin{array}{c}\text { Value of } I_{D}(\mathrm{eV}) \\
\text { from figure } 4\end{array}$ \\
\hline Zeaxanthin & 11628 & 5.51 & $5 \cdot 61$ \\
Lutein & 12500 & 5.62 & $5 \cdot 70$ \\
Fucoxanthin & 13333 & 5.72 & $5 \cdot 79$ \\
\hline
\end{tabular}

are $5.61,5.70$ and $5.79 \mathrm{eV}$ respectively. The $\bar{v}_{\mathrm{CT}}$ values used in figure 4 are for the complexes in solutions, whereas the $\bar{v}_{\mathrm{CT}}$ values used for the polyene complexes are in the solid state. The uncertainty involved in the values, however, is not more than $0 \cdot 1 \mathrm{eV}$.

Again assuming that $C_{1}$ values are essentially constant for similar type of complexes, the relationship between the ionization potential of two donors and the values of $\bar{v}_{\mathrm{CT}}$ with a common acceptor can be written as

$$
I_{D}(\text { donor- } 2)=I_{D}(\text { donor- } 1)+h v_{\mathrm{CT}}(2)-h v_{\mathrm{CT}}(1) \text {. }
$$

From the above, the value of ionization potential of donor- 2 can be estimated if the ionization potential of donor- 1 and the associated values of $\bar{v}_{\mathrm{CT}}$ are known. Assuming $I_{D}$ for $\beta$-carotene as $5.5 \mathrm{eV}$ (Vilesov 1960) and $v_{\mathrm{CT}}$ of $\beta$-carotene-iodine in the solid state complex as $11500 \mathrm{~cm}^{-1}$ (Mallik et al 1978), we have estimated the values of ionization potential of zeaxanthin, lutein and fucoxanthin. The ionization potential of polyenes estimated by two different methods are shown in table 1 for easy comparison. There is a difference of about $0.1 \mathrm{eV}$ between the two sets of values. Indeed figure 4 gives a value of $5.6 \mathrm{eV}$ for ionization potential of $\beta$-carotene as CT band is observed at $11500 \mathrm{~cm}^{-1}$. This value is $0.1 \mathrm{eV}$ higher than the reported value of $5.5 \mathrm{eV}$. This discrepancy may be due to a number of reasons. The assumption that the $C_{1}$ in (1) is constant may not be true for such wide range of ionization potential. Also the broad CT absorption bands of the polyene- $I_{2}$ complex makes $v_{C T}$ determination somewhat uncertain. Equation (2) gives better values of $I_{D}$ of polyenes as $C_{1}$ may be considered a constant for the small range of $I_{D}$ values for the polyenes studied.

It was observed that in addition to the charge-transfer bands on the longer wavelength side of the pure polyene bands, two new absorption bands appeared at about $366 \mathrm{~nm}$ and $295 \mathrm{~nm}$ in each complex. These were the well-known absorption bands of $I_{3}$ ion (Slifkin 1971a).

Polyenes are thus shown to form CT complex with iodine through the reaction

$$
\begin{aligned}
& \text { polyene }+I_{2}=\left[\text { Polyene... } I_{2}\right]=\left[\text { Polyene... } I^{+}\right]+I^{-} \\
& I_{2}-I^{-}=I_{3}^{-} .
\end{aligned}
$$

The new CT band at longer wavelength arises due to transition from the ground state to the excited state of the molecular complex. The molecular complex may then dissociate to give an inner complex [Polyene... $I^{+}$] and a negative iodine ion. The $I^{-}$ion can combine with free iodine to give $I_{3}^{-}$ion. The inner [Polyene... $I^{+}$] complex is expected to absorb at still longer wavelength (Ebrey 1967) where corresponding polymethines would absorb. But our experimental set up unfortunately does not permit us to record absorption spectra beyond $1000 \mathrm{~nm}$. 


\subsection{Photoconduction action spectra}

The photoconduction action spectra of pure polyenes and polyene-iodine complexes have been investigated. Two distinct photoaction bands, one at $320-450 \mathrm{~nm}$ and the other at $600-700 \mathrm{~nm}$ regimes are observed. Photoconduction action spectra of zeaxanthin and lutein and their complexes with iodine are shown in figures 5 and 6 respectively. In the pure polyenes, the long wavelength band is very intense whereas the $320-450 \mathrm{~nm}$ band is extremely weak. In the charge-transfer complex, on the other hand, the photoconduction peak at $320-450 \mathrm{~nm}$ region is very intense and the peak at $600-700 \mathrm{~nm}$ region appears to be very weak. Similar observation was reported for another polyene, crocetin in pure state ( $\mathrm{Pal}$ and Misra 1989). In pure polyenes, the high energy photoaction band has been attributed to the photoinjection of charge carriers from the electrode and the low energy band indicates direct electron-hole pair production. Close examination of CT absorption band and the photoconduction action band shows that they are not in the same spectral region. CT

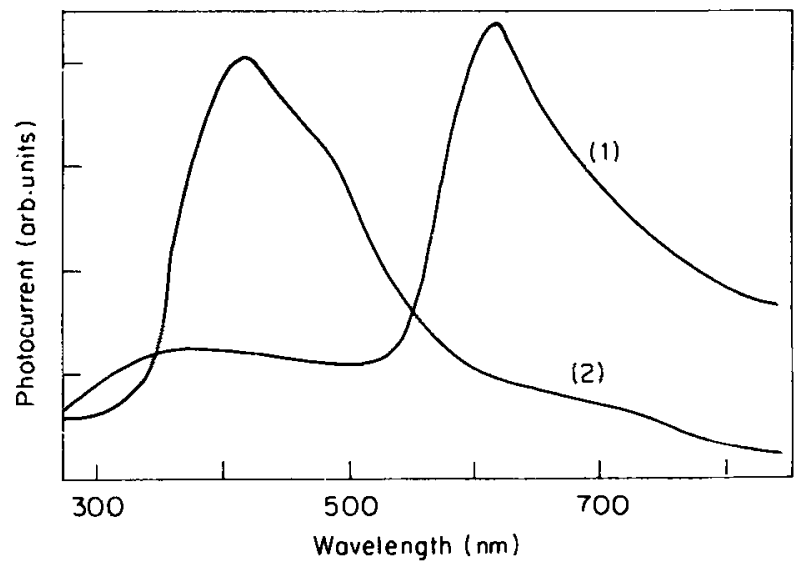

Figure 5. Photo action spectra for (1) zeaxanthin and (2) zeaxanthin-iodine complex.

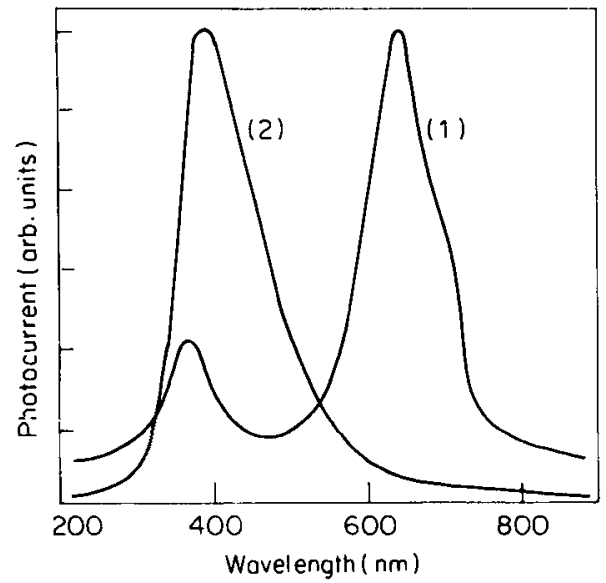

Figure 6. Photo action spectra for (1) lutein and (2) lutein-iodine complex. 
absorption band is at much lower energy (longer wavelength) for all the polyenes. The low energy photoconduction action band in the complex, though very weak, is in the same spectral region as in pure material. Similarly, the high energy band in the photoconduction spectrum, though very weak in pure materiai, is similar to the intense photoconduction band in CT complex. Thus the same two photogenerative events are operative both in pure polyenes and in polyene-iodine complexes but the principal contribution comes from different events in the two cases. This observation is at variance with that of Tamamura et al (1974) and Shimada et al (1973) with some other CT complexes. The shift of the photoconduction action spectra to lower energy from that of absorption spectra may be due to fast and efficient surface recombination in these materials. Only weakly absorbing light of longer wavelength penetrates the bulk material and generate photocarriers. Our results show that in pure polyenes the photocarriers are generated by direct electron-hole pair production, whereas in the complexes photoinjection of carriers from the electrode is mainly responsible for carrier generation.

\subsection{Dark and photoactivation energy}

In wide band-gap materials, as in organic solids, the localized states dominate the conduction process and the activation energy equations for extrinsic semiconduction as

$$
\begin{aligned}
& I_{d}=I_{o d} \exp \left(-E_{d}^{\prime} / k T\right) \\
& I_{p h}=I_{o p} \exp \left(-E_{p h}^{\prime} / k T\right)
\end{aligned}
$$

holds. In such materials either dominant electron levels or dominant hole levels contribute to the conductivity and the material behaves as extrinsic semiconductor. However, in some materials, both the electron and hole levels contribute equally and the material behaves as non-extrinsic semiconductor (Roberts and Schimdlin 1969). In such cases, half energy consideration is more realistic and $E_{d}^{\prime}=E_{d} / 2$, where $E_{d}$ is the measured thermal activation energy. This is the situation we encounter in dark conduction in pure polyenes. In photoconductivity different trap levels predominate and the extrinsic nature is retained.

In CT complexes, the donor material is doped with acceptor molecules and it is realistic to apply the conduction equation relevant to extrinsic material both for dark and photoconduction.

The Arrhenius plots for dark and photoconductivity of the polyenes and their CT complexes with iodine give thermal activation energy and the trapping levels. These are summarized in table 2. At room temperature $\left(27^{\circ} \mathrm{C}\right)$ both the dark and photoconductivity increases by several orders of magnitude but the ratio of photocurrent to dark current decreases on complex formation. This is a general observation for these types of complexes and are probably due to the formation of excess free thermal carriers in the dark or due to the thermal removal of electron from the donor to the acceptor leaving behind a hole into which other electrons may flow (Slifkin 1971 b). We observe that the dark activation energy decreases on complex formation for all the polyenes. But the photoactivation energy of all the polyenes increases due to the formation of polyene-iodine complex. All the activation energy data of different polyenes and their complexes are summarized in table 2 . 
Table 2. Dark current, photocurrent and activation energy of some polyenes and their CT complexes with iodine.

\begin{tabular}{lcccccc}
\hline Polyene & $E_{d}(\mathrm{eV})$ & $E_{d}^{\prime \mathrm{CT}}(\mathrm{eV})$ & $E_{p h}^{\prime}(\mathrm{eV})$ & $E_{p h}^{\prime \mathrm{CT}}(\mathrm{eV})$ & $I_{d}^{\mathrm{CT}} / I_{d}$ & $I_{p h}^{\mathrm{CT}} / I_{p h}$ \\
\hline Zeaxanthin & 2.18 & 0.50 & 0.27 & 0.50 & $7.98 \times 10^{4}$ & $2.25 \times 10^{4}$ \\
Lutein & 2.19 & 0.93 & 0.45 & 0.92 & $8.90 \times 10^{7}$ & $4.76 \times 10^{6}$ \\
Fucoxanthin & 2.98 & 1.51 & 0.38 & 1.52 & $2.08 \times 10^{5}$ & $1.10 \times 10^{4}$ \\
\hline
\end{tabular}

The important observation is that both dark and photoconductivity (when excited with white light) in the polyene-iodine complexes have the same value of activation energy. This property seems to be general with polyene-iodine complexes. Similar observation has been made in the case of some other CT complexes (Slifkin 1971a; Shimada et al 1973; Tamamura et al 1974). Identical activation energies for dark and photoconduction in CT complexes has often been thought to be due to heating effects of light (Eley et al 1975). However, appearance of an intense photoaction spectrum and its independence of light intensity suggests that in these polyene-iodine complexes the observed photoactivation is not due to the heating effect.

Trapping conduction mechanism can be used as the model to explain identical activation energy for dark and photoconduction in CT complexes. Charge carriers are trapped in the polyene-iodine complex below the conduction level which are thermally excited to the conduction band in the dark and the carriers thus generated can migrate through the crystal by repeated trapping-detrapping processes. The trap level depth below the conduction level is the energy required for trapping conduction which shows up as the thermal activation energy in the dark.

In the case of photoconduction in CT complexes, the photocarriers are injected into the conduction level from the electrodes by the absorption of light. The photocarriers thus generated can migrate through the crystal again by repeated trappingdetrapping processes as in the dark conduction. In this model, no activation energy is required for carrier generation and the activation energy observed is consumed in carrier migration.

An alternative mechanism of hopping conduction may also account for identical activation energy for dark and photoconduction. In the complex, carriers are generated spontaneously by photoirradiation or by thermalization (requiring very small amount of energy). The measured activation energy then is the hopping activation energy as the charge carriers migrate from one ion to another in the crystal. Unfortunately, the stacking arrangement for the molecules in the lattice is not known for these complexes to be definitive about the hopping route and the degree of charge transfer. Moreover, some of the measured activation energies are large enough for hopping conduction.

\section{Conclusions}

(i) Spectral studies confirm that the polyenes form molecular CT complexes with iodine in the solid state and that the ionization potential values of the polyenes estimated from charge-transfer absorption bands are quite reasonable.

(ii) Direct electron-hole pair production and photoinjection of carriers from the electrode are the two mechanisms of carrier generation in the pure polyenes and in 
their CT complexes, the former being the principal mechanism in pure material whereas the latter process predominates in the complexes.

(iii) Both dark and photoconductivity of polyenes increase by several orders of magnitude on CT complex formation but the ratio of photocurrent to dark current decreases in the complexes. Identical value of thermal activation energy of dark and photoconduction of the polyene-iodine complexes can be explained by trapping conduction mechanism, though hopping conduction of charge carriers cannot be ruled out.

\section{Acknowledgement}

A generous gift of the polyenes used in the present investigation by $\mathrm{M} / \mathrm{s}$ Hoffmann-LaRoche, Basel, Switzerland is thankfully acknowledged.

\section{References}

Chen E C M and Wentworth W E 1975 J. Chem. Phys. 633183

Ebrey T G 1967 J. Phys. Chem. 711963

Eley D D, Metelafi E and White M P 1975 J. Chem. Soc. Faraday I 71955

Foster R 1969 Organic charge transfer complexes (London and New York: Academic Press) p. 40

Kivelson S 1981 Mol. Cryst. Liq. Cryst. 7765

Mallik B, Ghosh A, Jain K M and Misra T N 1978 Indian J. Biochem. Biophys. 15233

Mallik B. Ghosh A and Misra T N 1979 Bull. Chem. Soc. Jpn 522092

Mallik B, Jain K M and Misra T N 1980 Biochem. J. 189547

Mark Index 1976 (ed.) Martha Windholz (New Jersey, USA: Mark \& Co. Inc.)

Murata I and Nakasuji K 1985 Mol. Cryst. Liq. Cryst. 12619

Pal P, Ghosh A and Misra T N 1988 J. Phys. Soc. Jpn 571006

Pal P and Misra T N 1989 J. Phys. D: Appl. Phys. 221358

Roberts G G and Schimdlin F W 1969 Phys. Rev. 180785

Sen S, Pal P and Misra T N 1993 J. Mater. Sci. 281367

Shimada M, Masuhara H and Matuga N 1973 Bull. Chem. Soc. Jpn 461903

Slifkin M A 1971 a Charge-transfer interactions of biomolecules (London and New York: Academic Press) p. 42

Slifkin M A 1971b Charge-transfer interactions of biomolecules (London and New York: Academic Press) p. 66

Tamamura T, Yasuba H, Okamoto K, Imai T, Kusabayashi S and Mikawa H 1974 Bull. Chem. Soc. Jpn 47448

Torrance J B 1985 Mol. Cryst. Liq. Cryst. 12655

Vilesov F I 1960 Dokl. Acad. Nauk. SSSR Ser. Mat. Fiz. 132632 